\title{
Analysis and evaluation of mobile rhythm games: game structure and playability
}

\author{
Doo Heon Song ${ }^{1}$, Kwang Baek Kim², Jong Hee Lee ${ }^{3}$ \\ ${ }^{1,3}$ Department of Computer Games, Yong-In SongDam College, Republic of Korea \\ ${ }^{2}$ Division of Computer Software Engineering, Silla University, Republic of Korea
}

\section{Article Info \\ Article history: \\ Received Jun 9, 2016 \\ Revised Nov 20, 2016 \\ Accepted Dec 11, 2016}

\section{Keywords:}

Mobile game

Note control

Playability

Rhythm Game

User interface

\begin{abstract}
The rhythm game is an action simulation game adapted to the presented music. While it is expected to have an educational effect as a functional game, the relationship between the operability and rhythm education under the mobile platform is still questionable. In Korea, it seems that mobile rhythm game is a minority maniac genre that are played mostly among teenagers and early twenties. In this paper, we select three mobile rhythm games that are most played by Korean gamers in analysis. First, we analyze the user interface layout, note control, evaluation style and level of difficulty for three games Deemo, Cytus, and Lanota. Then, we take a user survey in order to evaluate the playability of those games. All three games obtain high scores but there exist several statistically significant differences among games in analysis.
\end{abstract}

Copyright (C) 2019 Institute of Advanced Engineering and Science. All rights reserved.

\section{Corresponding Author:}

Doo Heon Song,

Department of Computer Games, Yong-In SongDam College, 571-1 Cheo-in Gu, Yong-in 17145, Republic of Korea. Email :dsong@ysc.ac.kr

\section{INTRODUCTION}

Rhythm game is designed to challenge a player's sense of rhythm and typically requires players to press buttons in a sequence dictated on the screen focus on dance or the simulated performance of musical instruments. With devices like PS2 or arcade-based equipment, the genre enjoys commercial successes with games such as Guitar Hero and RockBand series [1]. Since games in this genre can include physical movements in playing, it is used to the rehabilitation purpose [2] or early child motor skill training [3]. Furthermore, rhythm games are suggested to develop skills on how to use notes [4]. Also, it is reported that gamers of this genre have shown high level rhythm recognition skills on certain musical instrument through playing [5, 6]. The educational effect of this genre, however, is not clearly shown by a systematic review [7] but its intrinsic value on potential creativity learning is encouraged $[8,9]$.

In recent days, the game playing platform, especially for young teens who are the main customer of rhythm games, is moved to mobile devices and the value of rhythm game is then changed to pure entertaining purposes. Thus, the role of rhythm games in rehabilitative assistant of game-based learning is reduced. However, the importance of better user interface design is emphasized more than ever in game playing under mobile platform [10]. Hence, the usability of the music game is assessed by factors such as game interface, gameplay methodology and the game interaction mechanisms with the extended concept of 'playability' [11].

Game designers and scholars define the heuristics for testing game usability and playability somewhat differently [11-17] but overall, the playability of a game includes game interface, game play methodology and game interaction. Thus, the playability heuristics for rhythm games often include the user interface, the interaction between the musical tempo and screen positions to be hit, how the method of control and 
the program are linked, and how the game rules and the challenges encountered during the game and the feedback given $[11,14]$.

In this paper, firstly, we take a comparative analysis of mobile rhythm games that are played the most by Korean young gamers on user interface design, the control, and the level of difficulty. The basic user interface configuration on the mobile rhythm game consists of a score window, an energy bar, a background image and music as feedback on the note line, touch target, and play [4]. For our case, we analyze three leading mobile rhythm games in detail. Then we take the playability test by survey and see how well these games are accepted to Korean gamers

\section{ANALYSIS OF MOBILE RHYTHM GAMES}

A rhythm game is primarily played by comprehending the rhythm the game sets [12]. The player has to smash buttons in a rhythm provided by the game. As progress is made, the game successively increases the speed and complexity of the rhythms. Success means that the player's actions were close to the rhythm. Thus, success can be measured, assessed, and expressed in a score.

First, we select top three mobile rhythm games among Korean gamers with a survey conducted during June 2017 in the rhythm game communities. Among 112 respondents, 54\% were in their teens and 44\% were in 20's of age and 66\% were males. Subjects were playing 2.3 rhythm games in average and Deemo (86.6\%), Cytus $(62.5 \%)$, Lanota $(33.0 \%)$ are the most dominant games in playing Thus, our analysis is done for those three games. Coincidentally, all three games are made in Taiwan. There exist Korean mobile rhythm games but the market share is actually very small. Among major genre that young Korean gamers are playing investigated in [18], rhythm game players mostly enjoy casual RPG such as Maple story and Final Fantasy series from this pilot survey. This user group has quite different traits on game playing from horror-survival user group [19] or First-person-shooting user group [20]

\subsection{User interface and note control}

Deemo iterface is as shown in Figure 1. It is most common among rhythm games in that the note is sliding from top to bottom and the static non-movable evaluation line is at the bottom. The control of the note is only slide and click as shown in Figure 1(a).

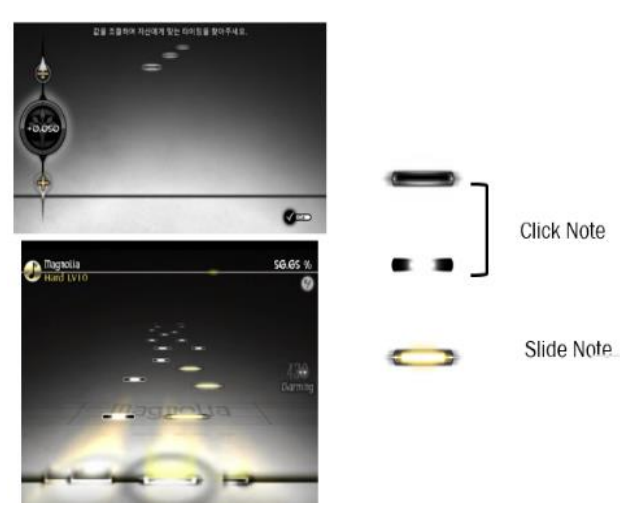

(a)

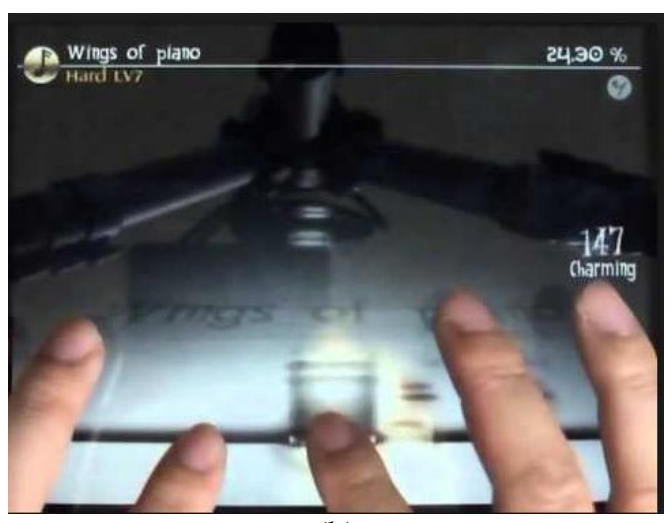

(b)

Figure 1. Deemo interface (a) Note control, (b) Playing scene

Cytus interface is as shown in Figure 2. It has moving evaluation line thus the gamer needs more attention in playing as shown in Figure 2(b). Cytus adds "hold" control so that the user can efficiently response to the moving evaluation line. Figure 2(a) shows click, drag, and hold in the clockwise order from upper left side.

Figure 3 shows the Lanota interface. Lanota has more note control and rotating evalustion disk (not a line). The note control is devided into click, flick that needs control to a certain direction of note shape, rail (sliding hold), and catch (a.k.a. slide) as shown in Figure 3 in clockwise order from upperleft side. Such differences of user interface and note control make different type of user behaviour and different level of difficulty design. Table 1 summarizes the difference of playing style among those three games. 

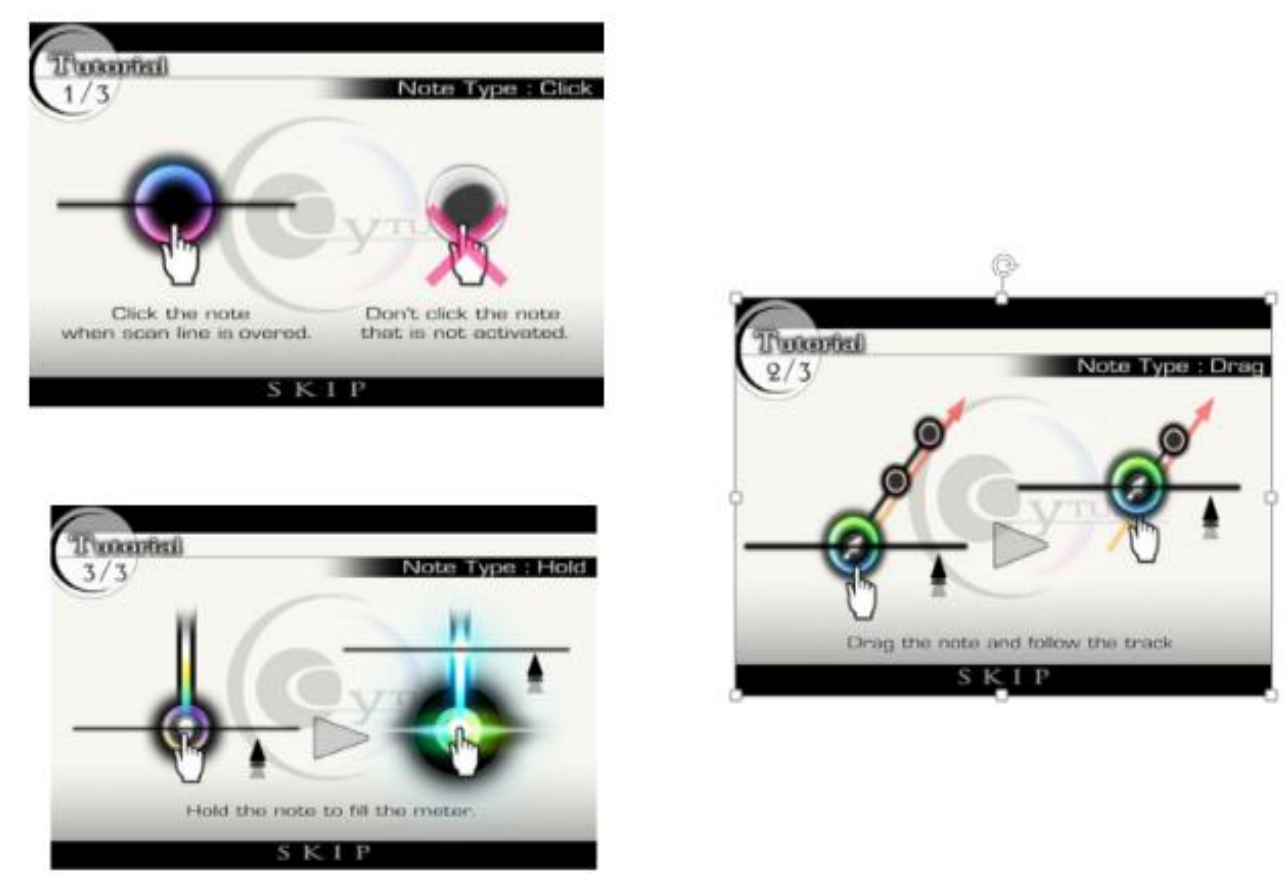

(a)

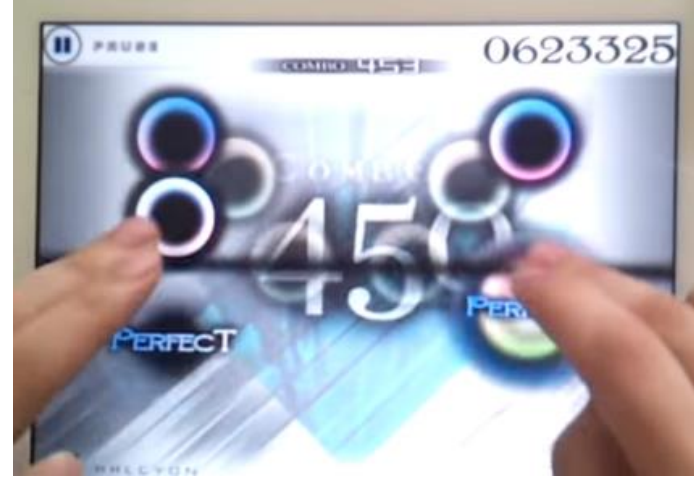

(b)

Figure 2. Cytus interface (a) Note control, (b) Playing scene
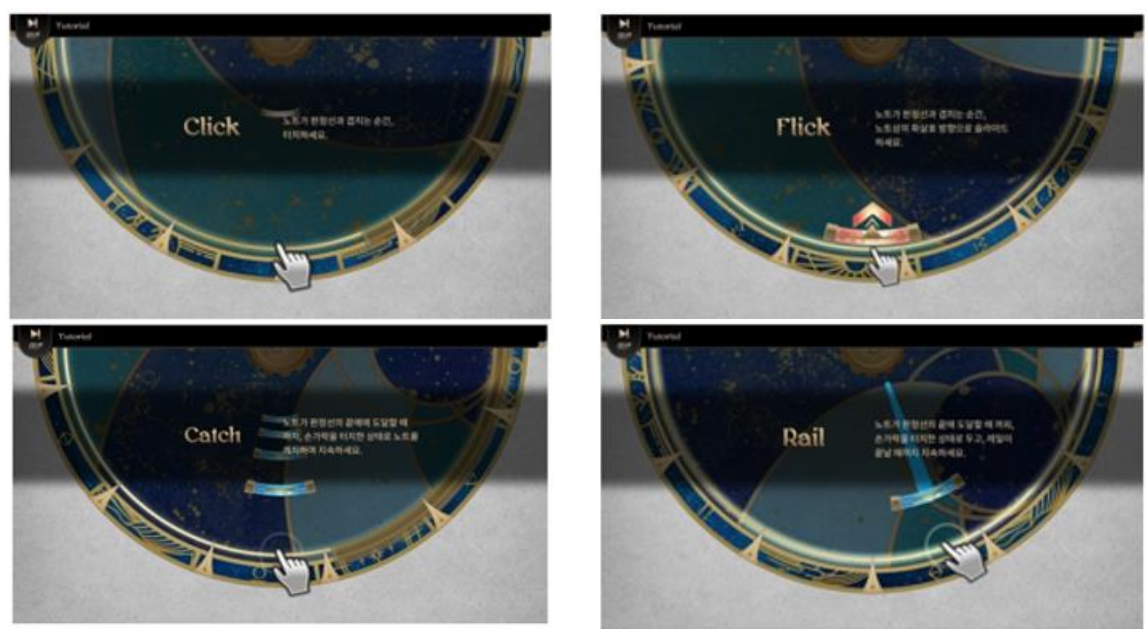

Figure 3. Lanota interface 
Table 1. Game features of mobile rhythm games

\begin{tabular}{cccc}
\hline Game/Feature & Deemo & Cytus & Lanota \\
\hline Level of Difficulty & Set before selecting music & Set before selecting music & Set after selecting music \\
Note Speed Option & Can Initialize & None(Static) & Can Initialize \\
Map & Yes & No & Yes \\
Different Chapter & Yes & Yes & Yes \\
Ranking & No & No & No \\
\hline
\end{tabular}

\subsection{Level of difficulty}

The level of difficulty in rhythm games tends to be proportional to the number of notes in the music but not absolutely. Rhythm games have more diverse control in arcade platform than in the mobile platform. Under mobile platform, the user interface is quite constrained thus much less things are to be controlled. As a result, the level of difficulty in mobile platform is lower than that of arcade rhythm games. Still, three target mobile rhythm games have different modes and levels of difficulty. There exists a quantitative tendency of the number of notes per music in play with respect to the levels of difficulty as shown in Table 2.

Table 2. Level of difficulty as function of notes per music

\begin{tabular}{ccccccc}
\hline Game & \multicolumn{5}{c}{ Difficulty } & \multicolumn{4}{c}{ Levels } & Mode \\
\hline Deemo & 278.6 & 527.0 & 757.8 & 12 & 3 \\
Cytus & 528.3 & \multicolumn{7}{c}{727.0} & 9 & 2 \\
Lanota & 310.2 & 473.9 & 646.4 & 858.5 & 15 & 4 \\
\hline
\end{tabular}

Deemo has three modes (easy, normal, and hard) with 12 different levels of difficulty. Cytus has only easy and hard mode with total 9 levels. Lanota has four modes - whisper, acoustic, ulrea, and master - with total 15 levels. As noted earlier, the number of notes per music is not the absolute factor to decide the perceived difficulty . The mixture of different notes, evaluation method (static/moving line/disk), and note control actions all contribute to form the level of perceived difficulty.

\section{PLAYABILITY EVALUATION BY SURVEY}

Evaluation with traditional 'usability' heuristics, that is an expert-based usability inspection methods for software development [21], cannot be directly applied to the evaluation of video games because application software and video games are very different in context and usability heuristics do not cover all aspects of gaming such as fun, entertainment, and enjoyment [14].

The seminal work done by Korhonen and his group [11] proposed 29 heuristics divided into three categorize as follows: 'Game-Usability', 'Game-Mobility' and 'Game-Play' [14]. However, their heuristics is designed for games played in general mobile platform thus it is too general to be applied to a specific genre. For example, people devise their own set of heuristics for management simulation genre [22] and casual mobile games [23] for usability test.

For an effort to test the playability of rhythm games, Chen \& Lo's work [15] defines their own set of heuristics that are divided into another three categories - functional, structural, and audiovisual - with 14 heuristics. Their analysis showed that music rhythm game interface design includes level information and visual design representing musical beat and that should be included in the heuristics.

Thus, in this paper, we will use Chen \& Lo's heuristic as a standard set but we find that many of our subjects in pilot study were confused in several questions of their original set of heuristics. Thus, we eliminate 4 questions from their set of heuristics as shown in Table 3 and added 4 other questions that Korean gamers are sensitive in evaluating the functionality of game interface as question number 11 to 14 of Table 4.

The survey was done in November 2017 and subjects are chosen from rhythm game communities. Selected subjects are long time users (at least 3 months) who are playing at least one game of our three games in analysis at the time of survey. Unfortunately, with such qualification of subjects for playability evaluation, the demographic aspects of subjects were not well controlled. There are 25 Deemo users, 9 Cytus users, and 5 Lanota users. Among those 39 subjects in this survey, only 7 are males (18\%). However, the age group distribution is almost equal in that there are 20 teens and 19 others are in their 20's. For this research, a "5-point" Likert Scale was used to determine the acceptance of users towards mobile game features, ranging from "Strongly Agree" (5), "Agree" (4), "Neutral" (3), "Disagree" (2) and "Strongly Disagree" (1). 
Table 3. Omitted questions from [15] Omitted questions

Does the game rating allow me to have a sense of accomplishment?

Do you feel excited when playing the game?

Did you like the rhythm of the music?

Did you feel excited by the visual effects?

Table 4. Playability questionnare result

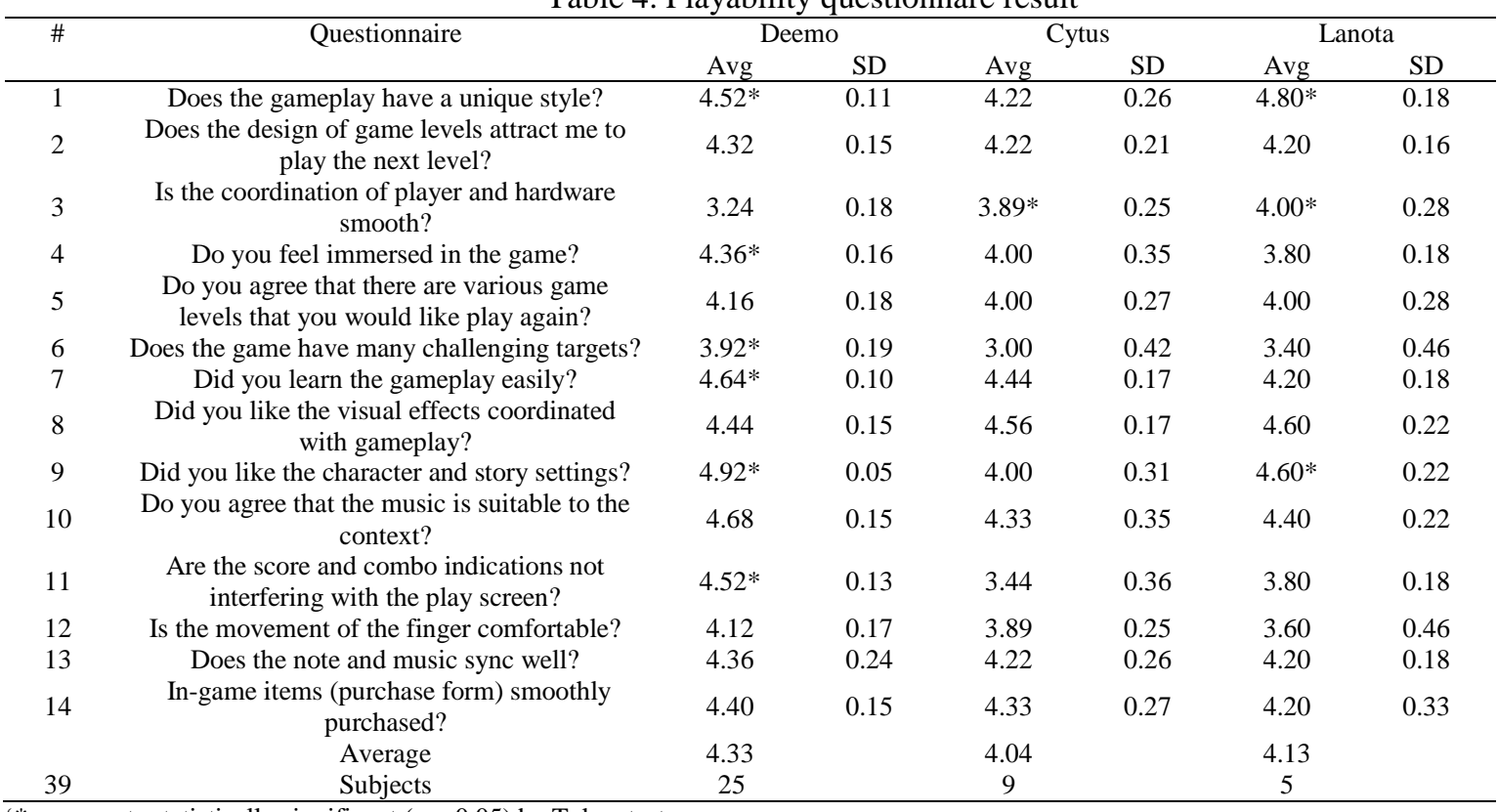

** represents statistically significant $(\mathrm{p}<0.05)$ by Tukey test

In analysus, we found that all three games are well accepted by Korean young gamers as a whole such that all games scored over 4.0 in average 5 point Likert scale from the 14 questions. Specifically, subjects gave highest uniqueness to Lanota as expected and Deemo is the highst in evaluation in feeling immersion (Q4), challenging targets (Q6), easy rule understanding (Q7), character \& story setting (Q9), non-interfering layout (Q11) but is least welcomed in player/hardware coordination(Q3). Cytus shows good player/hardware coordination $(\mathrm{Q} 3)$ but is least unique style $(\mathrm{Q} 1)$, least challenging (Q6), less immersive (Q4), unfavored character \& story (Q9), and most screen interference (Q11). Lanota obtains also the highest evaluation in smooth hardware/player coordination (Q3) and good character \& story setting (Q9) but subjects feel that lanota is game that is the least immersive (Q4), less challenging $(\mathrm{Q} 6)$, the hardest to understand rules of playing (Q7), and has screen interference (Q11). Overall, rhythm games analyzed in this paper are less welcomed in smooth hardware/player coordination (Q3), challenging targets (Q6), and finger movement (Q12).

\section{CONCLUSION}

Mobile rhythm game is a maniac game among young Korean gamers who like the music playing. Rhythm games usually do not have any guild system nor serious battles/competitions that are typical characteristics of mainstream games which young Korean gamers play. Such maniac genre gamers have different behaviour patterns and shoyld be analyzed carefully such as this rhythm gamers and Chinese Fleet Moe gamers [24]. Although the game has its own story such as an adventure to find the chaotic notalium that is the key element to make the world in peace (Lanota) or upbringing the memory with music to rescue the world from the virus attack(Cytus), the story is not the main reason to play. Touching the sliding note is the main activity in the game and the story proceeds with collecting items or raising up the character after such note clicking.

In this paper, we analyze three most popular mobile rhythm games among Korea young gamersDeemo, Cytus, and Lanota - on the user interface layout, note control, evaluation line setting and other game options. Heuristic evaluation of playability based on previous research[15] plus several added questions shows that all three games are in general welcomed. However, there are discrepancies in feeling immersive, understanding game playing, character \& story setting sarisfaction etc.. Overall, rhythm games analyzed in this 
paper shows high (over 4.0 out of 5.0) score except in smooth hardware/player coordination, challenging targets, and finger movement. Such result may lead mobile rhythm game designers to focus on better user playability with adquate control in interface design.

\section{REFERENCES}

[1] Miller K., "Schizophonic performance: Guitar hero, rock band, and virtual virtuosity," Journal of the Society for American Music, 3 (4), 395-429, 2009.

[2] Yuan B, Folmer E., "Blind hero: enabling guitar hero for the visually impaired," In Proceedings of the 10th international ACM SIGACCESS conference on Computers and accessibility, ACM, 2008, 169-176.

[3] Umanski D, Kogovšek D, Ozbič M, Schiller NO, "Development of a voice-based rhythm game for training speech motor skills of children with speech disorders," In Proceedings 8th International Conference Disability, Virtual Reality and Associated Technologies, 255-262, 2010.

[4] Park SI, Kihl TS, "Rhythm game design for effective music education," Journal of Korea Game Society, 12(1), 33-42, 2012.

[5] Pasinski AC, Hannon EE, Snyder JS, "How musical are music video game players?," Psychonomic bulletin \& review, 23(5), 1553-8, 2016.

[6] Nouwen M, Schepers S, Mouws K, Slegers K, Kosten N, Duysburgh P, "Designing an educational music game: What if children were calling the tune?," International journal of child-computer interaction, 1(9), 20-32, 2016.

[7] Bégel V, Di Loreto I, Seilles A, Dalla Bella S, "Music games: potential application and considerations for rhythmic training, Frontiers in human neuroscience," 11(online), 2017.

[8] Roesner D, Paisley A, Cassidy G, "Guitar Heroes in Music Education?: Music games and their potential for musical and performative creativity, In Music Video Games: Performance, Politics, and Play, Austin, M. Editor, Bloomsbury Publishing USA," 197- 228, 2016.

[9] Jenson J, De Castell S, Muehrer R, Droumeva M, "So you think you can play: An exploratory study of music video games," Journal of Music, Technology \& Education, 9(3), 273-288, 2016.

[10] Ji Z, Huang WH, Zhang X, "Design and implementation of a game interface interaction on smartphone," Journal of Intelligent \& Fuzzy Systems, 34(2), 2018:923-931.

[11] Korhonen H, Koivisto E. "Playability heuristics for mobile games, " In Proceedings of the 8th conference on Humancomputer interaction with mobile devices and services, ACM, 9-16, 2006.

[12] Pichlmair M, Kayali F, "Levels of Sound: On the Principles of Interactivity in Music Video Games," In DiGRA Conference 2007.

[13] Paavilainen J., "Critical review on video game evaluation heuristics: social games perspective," In Proceedings of the International Academic Conference on the Future of Game Design and Technology, ACM, 56-65, May, 2010.

[14] Soomro S, Ahmad WF, Sulaiman S., "Evaluation of mobile games using playability heuristics" In International Visual Informatics Conference, 264-274, Nov 2013.

[15] Chen, CH., Lo, CS, "The development of a music rhythm game with a higher level of playability," In Advanced Materials for Science and Engineering (ICAMSE)," International Conference on, IEEE, 132-5, 2016.

[16] Hsu FC. "User Satisfaction and System Environment Convenience for Interface Design of Mobile Games," Ekoloji, 27(106), 1211-1215, 2018.

[17] Chen YC, Li SR. "Cognition difference between players of different involvement toward the concrete design features in music games," PloS one, 14(5), (online)e0216276, 2019.

[18] Song DH, Park S, Yang SW, Yang Y, Won K. Gender Differences and Gender Stereotype in Play Style among Young Korean Gamers. Journal of the Korea Institute of Information and Communication Engineering, 21(1): 72-81, 2017.

[19] Song DH et al. From Agasa Cristie to group image play - Analysis of horror survival game panic room: Escaping from the den on emotional elements development. International Journal of Electrical and Computer Engineering, 81(2):644-650, 2018.

[20] Song DH et al. Comparative analysis of first person shooter games on game modes and weapons-military-themed, overwatch, and player unknowns' battleground. Indonesian Journal of Electrical Engineering and Computer Science, 13(1):116-122, 2019.

[21] Nielsen J, Mack RL. Usability Inspection Methods, New York. John Wiley \& Sons, 1994.

[22] Oh YB, Kim WK, "A Study on Mobile Game UI Design Element Considering User Experience -Focused on Management Simulation Game," Journal of Korean Society of Design and Culture, 23(2); 413-428, 2017.

[23] Zhao L, Kim HH. "Research on usability of mobile casual game interface - Focusing on top three casual game in Korean Google play," Journal of Digital Design, 13(4); 361-371, 2013.

[24] Song, DH. "Analysis of Korean Gamers' Preferences on Chinese Mobile Games," Journal of the Korea Institute of Information and Communication Engineering, 22(7), 935-942, 2018. 


\section{BIOGRAPHIES OF AUTHORS}

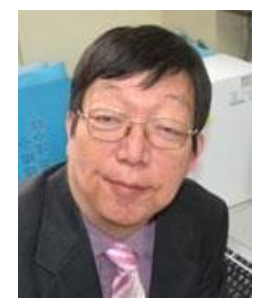

Doo Heon Song received his B.S. from Seoul National University in 1981 and M.S. from the Korea Advanced Institute of Science and Technology in 1983 in Computer Science. He received his Ph.D. Certificate in Computer Science from the University of California at Irvine in 1994. He has been a professor at Department of Computer Games, Yong-in Songdam College, Korea, since 1997. His research interests include machine learning, artificial intelligence, fuzzy systems, machine learning, medical image processing, and computer game design. He is currently the associate editor of Journal of Information and Comunication Convergence Engineeing.

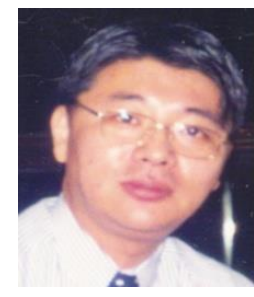

Kwang Baek Kim received his M.S. and Ph.D. degrees from the Department of Computer Science, Pusan National University, Busan, Korea, in 1993 and 1999, respectively. From 1997 to the present, he is a professor at the Department of Computer Engineering, Silla University, Korea. He is currently an associate editor for Journal of Intelligence and Information Systems and The Journal of Information and Communication Convergence Engineering. His research interests include fuzzy clustering and fuzzy control system, data mining, image processing, and bioinformatics.

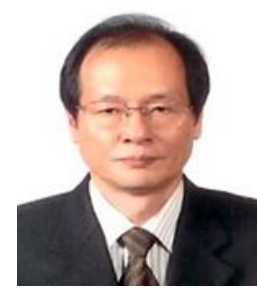

Jong Hee Lee received his B.S., M. S., Ph. D. In Electrical Engnieering from KyungBook University in 1978, 1984, 1990 in respectively. He has been a professor at Division of Computer Software Engineering, Sillla University, Busan, Korea since 1988. His research area includes intelligent system control, computer science education, and agent system. 